\title{
Performance Evaluation of Active Windows Using Speech Intelligibility
}

\author{
Wongeun $\mathrm{Oh}^{1}$
}

\begin{abstract}
This paper presents an experimental work on the performance assessment of windows equipped with the active noise control technology. Recently, several active windows have been studied and proved to reduce the transmitted exterior noise through an open window. However, the performance of the systems has been evaluated only in terms of the sound pressure level or the power spectrum. In this paper, the speech intelligibility is adopted to assess the performance of the active windows for more practical measurements. The results of scale-model experiments show that the active window can improve the intelligibility as well as reduce the noise level.
\end{abstract}

Keywords - Active Noise Control, active windows, STI, speech intelligibility

\section{INTRODUCTION}

$\mathrm{T}$ HE traffic noise is one of the major environmental problem that has effects on health and living quality in the cities. The external noises can be effectively reduced by closing windows. However, this solution is less effective in summer times or in southern countries where the windows need to be frequently opened. Several active noise control (ANC) systems have been applied to the open windows to solve this problem [1]-[3]. The systems were proved to reduce the transmitted exterior noise when the windows were open. Huang investigated the active window system that was constructed by staggering the opening sashes of a spaced double glazing window to allow ventilation and natural light [1]. Kwon proposed an active window system that consisted of reference sensors installed outside the room and control sources installed on the window frame. The performance of the system was verified in a scale-model experiment, and the results showed that the overall average sound pressure level inside the scale model was reduced by as much as $10 \mathrm{~dB}$ [2]. Pàmies presented an experimental work on active control of sound transmission through a restricted opening bottom hinged window and its application to aircraft fly-over incident noise. The result showed that an increase of almost $3 \mathrm{~dB}$ of transmission loss in the low frequency range [3].

In the above works, the performance of the active windows has been evaluated in terms of the sound pressure level or the power spectrum of the reduced noise. However, the effects of the active windows on the speech intelligibility and the clarity in the rooms are not studied yet. In general, the hearing perception of human depends not only on the sound level but

Wongeun $\mathrm{Oh}^{1}$ is with Sunchon National University, Suncheon-si, Jeonnam, Korea also on the other factors - spectrum of the noise, masking, and room acoustics, hence more practical measures are necessary to assess the performance of the active window.

In this paper, the speech intelligibility is considered and tested to assess the performance of the active window systems. In the experiment, a duct-type active window system is used and three intelligibility parameters(STI, \%Alcons, and C50) are evaluated. The results show that the active window can improve the intelligibility in the rooms.

\section{METHODOLOGY}

\section{A. Intelligibility parameters}

The intelligible speech means hearing the consonants and vowels correctly to identify the words and sentence structure. Several criteria have developed and used to measure the speech intelligibility. In room acoustics, STI(Speech Transmission Index), \%Alcons(Percentage Articulation Loss of Consonants), and C50 are one of the most frequently used parameters. The subjective scale of the parameters is in Table 1 [4].

TABLE 1 SUBJECTIVE QUALITY OF THE STI AND \%ALCONS

\begin{tabular}{lll}
\hline \hline \multicolumn{1}{c}{ Quality } & \multicolumn{1}{c}{ STI } & \multicolumn{1}{c}{$\%$ Alcons } \\
\hline Excellent & $0.75-1$ & $0 \%-3 \%$ \\
Good & $0.6-0.75$ & $3 \%-7 \%$ \\
Fair & $0.45-0.6$ & $7 \%-15 \%$ \\
Poor & $0.3-0.45$ & $15 \%-33 \%$ \\
Bad & $0-0.3$ & $33 \%-100 \%$ \\
\hline
\end{tabular}

\section{B. The duct-type active window systems}

A duct-type active window system is selected to test the effects on the intelligibility. The duct-type active windows have advantages than the other active windows in that its simple structure and control algorithm [5]. A cross section sketch of the proposed system installed in a room is shown in Fig. 1. The ANC window consists of a short length duct, a reference microphone, an error microphone, and a control loudspeaker. The system makes the destructive interference between the incident noise and the control sound, and the sound pressure levels caused by the exterior noise passing through the window can be decreased. 


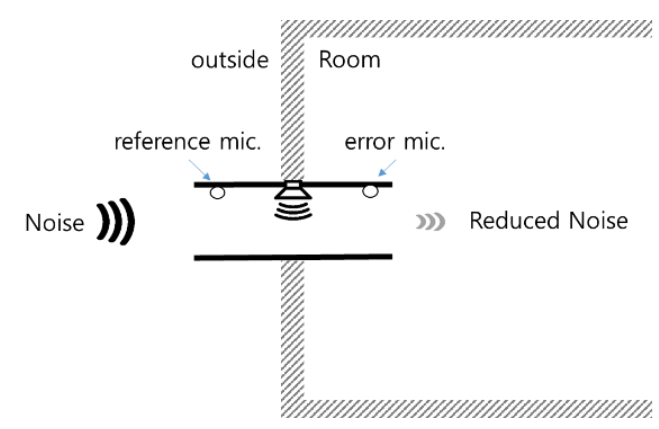

Fig. 1 Sketch of the proposed ANC window

\section{Model room}

Fig. 2 shows the structure and dimension of the model room used for the experiments. The dimension of the room is $0.9 \mathrm{~m} \mathrm{x}$ $1.2 \mathrm{~m} \times 0.9 \mathrm{~m}$. The room is made of $3 \mathrm{~cm}$ thick PVC panels and the walls are covered with $2.5 \mathrm{~cm}$ thick sound absorbing materials. The dimension of the window is $0.12 \mathrm{~m} \mathrm{x} 0.19 \mathrm{~m}$, and the duct type active window(length $0.52 \mathrm{~m}$ ) is installed on it.

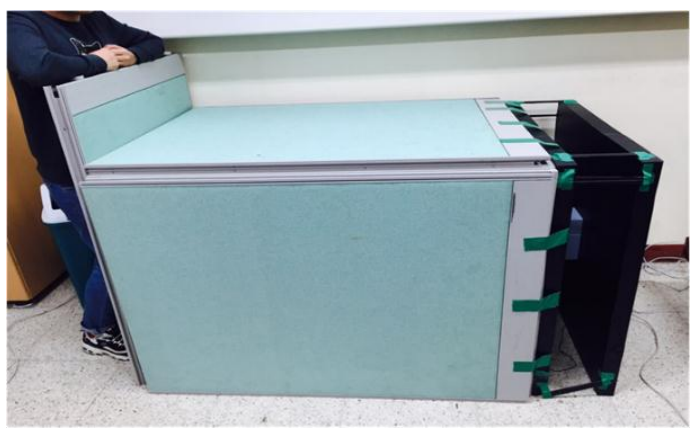

(a)

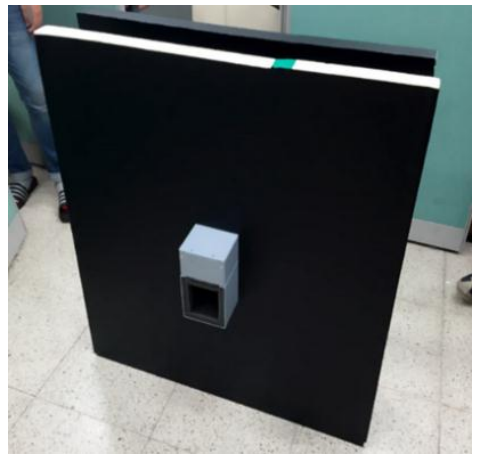

(b)

Fig. 2 Structure of the model room (a) side view (b) front view

As for a duct-type window, a 'Demo S-Fan 90' ANC system by Silentium is adopted [6]. It consists of two microphones, two loudspeakers, an adaptive controller, and two software tools. The Monitor program is used to interface between the laptop and the controller, and the S-ANC Tool is for analyzing the data acquired during the calibration. Fig. 3 shows a photograph of the Demo S-Fan.

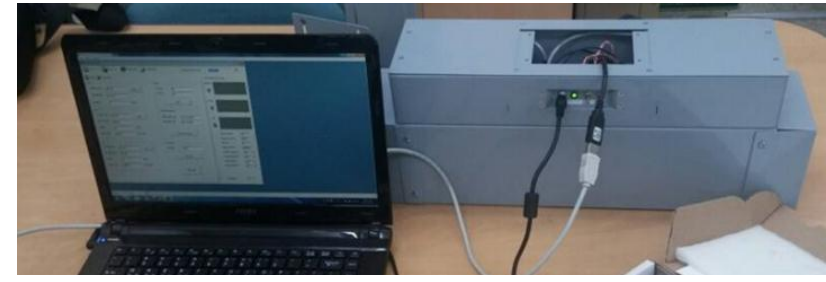

Fig. 3 Photograph of the Demo S-Fan

\section{D.Experimental procedure}

A loudspeaker located outside the room generated pink noise to simulate the exterior noise. A measurement microphone (Earthworks M30S) is used to pick up the interior noise of the room and EASERA software is used for the intelligibility analysis.

\section{E. Results}

Table 1 lists the measurement values of three intelligibility parameters. The intelligibility is improved when the ANC is ON.

TABLE 2

COMPARISON OF THE INTELLIGIBILITY PARAMETERS

\begin{tabular}{llll}
\hline \hline & \multicolumn{1}{c}{ ANC OFF } & \multicolumn{1}{c}{ ANC ON } & Difference \\
\hline STI & 0.477 & 0.5 & +0.023 \\
Alcons $(\%)$ & $12.9 \%$ & $11.3 \%$ & $+1.6 \%$ \\
C50 & $-1.5 \mathrm{~dB}$ & $-0.6 \mathrm{~dB}$ & $+0.9 \mathrm{~dB}$ \\
\hline \hline
\end{tabular}

In the experiment, the averaged SPL difference is $6.4 \mathrm{dBA}$ between ANC ON and OFF. The spectrum of the noise measured in the room is shown in Fig. 4.

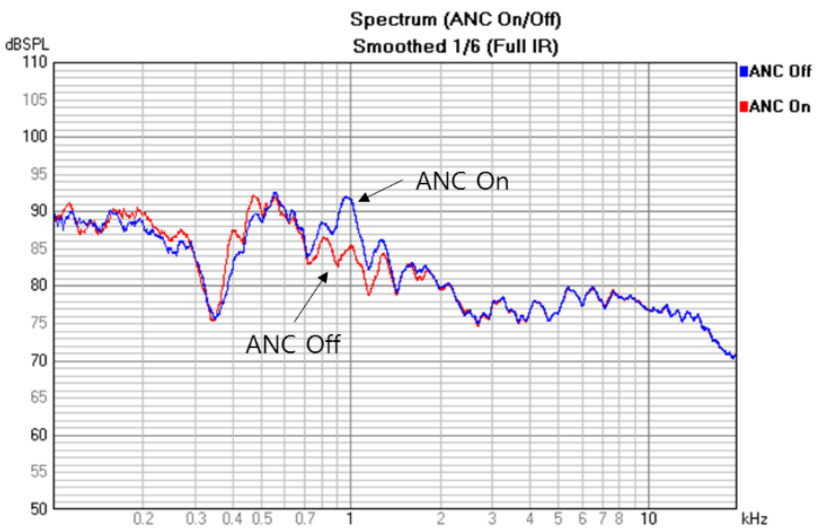

Fig. 4 Averaged spectrum at the monitor microphone with and without ANC.

\section{DISCUSSION AND CONCLUSION}

In this paper, the performance of the duct-type active windows has been evaluated in terms of the speech intelligibility parameters as well as the sound pressure level and 
the power spectrum. The hearing perception of human depends not only on the sound level but also on the other factors i.e. spectrum of the noise, masking, and room acoustics. Hence, the intelligibility measure is more suitable to assess for the subjective quality of the active windows.

Three intelligibility measures(STI, \%Alcons, and C50) have been tested in the scale-model experiments. The results show that the active window can improve the intelligibility as well as reduce the noise level in the room.

\section{ACKNOWLEDGMENT}

This research was supported by Basic Science Research Program through the National Research Foundation of

Korea(NRF) funded by the Ministry of
Education(NRF-2014R1A1A2057077).

\section{REFERENCES}

[1] H. Huang, X. Qiu, and J. Kang, "Active noise attenuation in ventilation windows," J. Acoust. Soc. Am., vol. 130, no. 1, pp. 176-188, Jul. 2011. http://dx.doi.org/10.1121/1.3596457

[2] B. Kwon and Y. Park, "Interior noise control with an active window system," Appl. Acoust., vol. 74, no. 5, pp. 647-652, May 2013. http://dx.doi.org/10.1016/j.apacoust.2012.11.005

[3] T. Pàmies, J. Romeu, M. Genescà, and R. Arcos, "Active control of aircraft fly-over sound transmission through an open window," Appl. Acoust., vol. 84, pp. 116-121, 2014. http://dx.doi.org/10.1016/j.apacoust.2014.02.018

[4] G. Ballou, Handbook for sound engineers, 4th ed. Focal Press, 2008.

[5] W. Oh, "Noise reduction in rooms using a single channel active noise controller," in Proc. Engineering \& Technology, Computer, Basic \& Applied Sciences, 2016, to be published.

[6] Silentium LTD., "Silentium's Demo S-Fan 90." [Online]. Available: http://www.silentium.com/products/demo-s-fan-90/. 